\title{
Variação floral e biologia reprodutiva de Manettia cordifolia Mart. (Rubiaceae)
}

\author{
HÉLDER CONSOLARO ${ }^{1,3}$, ELAINE BENTO DA SILVA² e PAULO EUGÊNIO DE OLIVEIRA ${ }^{1}$
}

(recebido: 19 de março de 2004; aceito: 5 de novembro de 2004)

\begin{abstract}
Floral variation and reproductive biology of Manettia cordifolia Mart. (Rubiaceae)). Rubiaceae is the largest family with distylous species among the Angiosperms. The distyly in those species may vary in form and degree leading to homostyly, monomorphism and dioecy. The objective of this study was to describe the floral biology, pollination and breeding system of Manettia cordifolia Mart. The study was carried out in The Panga Ecological Station and riverside of the Uberabinha (Uberlândia, MG), and complemented with observations of collections deposited in herbaria. Manettia cordifolia is a vine with flower traits of typical of hummingbird pollination syndrome. The main pollinator was the hummingbird Phaetornis pretrei. The plant cannot be considered a distylous species, although it is in a genera known as distylous. The observed characteristics indicate a species with pin-monomorphy, probably derived from a distylous ancestral. Controlled pollinations showed a selfincompatible and non-apomictic species. Pollen tube growth down to the ovary after selfing is similar to the observed in pin pistils of truly distylous congeneric species. The absence of information on the distyly genetic control in the Rubiaceae makes it difficult to interpret the variations observed on the present study.
\end{abstract}

Key words - distyly, heterostyly, monomorphism, pollination, self-incompatibility

RESUMO - (Variação floral e biologia reprodutiva de Manettia cordifolia Mart. (Rubiaceae)). Rubiaceae é a maior família que contém espécies distílicas dentre as Angiospermas. A distilia nessas espécies pode apresentar variações de diferentes formas e graus, originando derivações como homostilia, monomorfia e dioicia. Este estudo tem objetivo de descrever a biologia floral, a polinização e o sistema reprodutivo de Manettia cordifolia Mart. O estudo foi realizado na Estação Ecológica do Panga e margens do Rio Uberabinha (Uberlândia, MG) e complementado com observações de material botânico depositado em herbários. Manettia cordifolia é uma liana com flores tipicamente ornitóflias. Seu polinizador principal foi o beija-flor Phaetornis pretrei. A espécie não foi considerada distílica, apesar de estar dentro de gênero considerado distílico. Suas características indicam ocorrência de monomorfismo longistílico, provavelmente derivado de um ancestral distílico, não sendo encontrados indivíduos brevistilos. Os testes de polinizações controladas indicam que a espécie é auto-incompatível e não apomítica. O crescimento do tubo polínico até o ovário após autopolinização é similar ao observado em flores longistilas de espécies verdadeiramente distílicas. A interpretação dos resultados é dificultada pela ausência de informações sobre o controle genético da heterostilia nas Rubiaceae.

Palavras-chave - auto-incompatibilidade, distilia, heterostilia, monomorfismo, polinização

\section{Introdução}

Heterostilia é um tipo de polimorfismo floral geneticamente controlado e encontrado em aproximadamente 28 famílias de Angiospermas, sendo interpretado como um mecanismo que promove a polinização cruzada (Barrett et al. 2000, Li \& Johnston 2001). Espécies heterostílicas apresentam flores com hercogamia recíproca, juntamente com um sistema genético de incompatibilidade, no qual apenas cruzamentos intermorfos resultam em formação de

1. Universidade Federal de Uberlândia, Instituto de Biologia, Caixa Postal 593, 38400-902 Uberlândia, MG, Brasil.

2. Faculdade de Medicina do Triângulo Mineiro, Programa de Pós-Graduação em Medicina Tropical e Infectologia, Uberaba, MG, Brasil.

3. Autor para correspondência: hnconsolaro@hotmail.com frutos (Ganders 1979, Bawa \& Beach 1983, Kohn \& Barrett 1992, Thompson \& Dommée 2000). A hercogamia recíproca manifesta-se na apresentação de morfos florais que são diferenciados pela altura do estigma e posicionamento recíproco das anteras, podendo ocorrer em condições distílicas e tristílicas (Ganders 1979). Plantas distílicas são distinguidas por apresentarem indivíduos cujas flores possuem estiletes longos e estames curtos (flores longistilas) e indivíduos com flores de estiletes curtos e estames longos (flores brevistilas) (Ganders 1979). Os mecanismos que regem o heteromorfismo floral e o sistema de incompatibilidade são fortemente relacionados, devido à existência da ligação entre os loci gênicos que os controlam, formando o que é chamado de supergene (Ganders 1979).

Variações na apresentação da heterostilia podem ocorrer tanto entre gêneros quanto entre indivíduos ou populações, dentro de espécies tipicamente distílicas, 
além de poderem estar presentes entre populações distílicas separadas por barreiras geográficas ( Li \& Johnston 2001). Essas variações podem ocorrer por vários motivos, tais como situações desfavoráveis à polinização cruzada por meio de ineficiência ou ausência de polinizadores, ou ainda, por reprodução clonal, perturbações ambientais e espécies com algum grau de autocompatibilidade (Sobrevila et al. 1983) provinda do "cross-over" ou mutação do supergene que controla a distilia (Li \& Johnston 2001).

Rubiaceae contém o maior número de espécies distílicas dentre todas as famílias nas quais tal dimorfismo é conhecido, sendo encontrada em 416 espécies distribuídas em 91 gêneros (Ganders 1979, Barrett \& Richards 1990, Passos \& Sazima 1995). A ocorrência de variações na apresentação da distilia é comum na família, sendo demonstrada para espécies de Psychotria, Palicourea, Bouvardia e Guetarda (Sobrevila et al. 1983, Hamilton 1990, Richards \& Koptur 1993, Faivre \& McDade 2001, Coelho \& Barbosa 2003).

Mesmo possuindo flores com corola gamopétala e relativamente especializadas, as Rubiaceae apresentam um amplo espectro de polinizadores (Robbrecht 1988, Barrett \& Richards 1990). Suas flores tubulares favorecem visitantes com aparelho bucal longo, sendo mais comumente polinizadas por borboletas e abelhas com probóscide longa (Taylor 1996, Stone 1996, Ortiz et al. 2000, Castro \& Oliveira 2002) ou freqüentemente por mariposas (Imbert \& Richards 1993, Wolff et al. 2003) e moscas (Riveros et al. 1995, Ortiz et al. 2000). Trabalhos mostrando visitas de beija-flores em espécies de Rubiaceae são comuns, como registrado para Psychotria, Manettia, Palicourea, Cephaelis, Hamelia e Ferdinandusa (Bawa \& Beach 1983, Snow \& Snow 1986, Feinsinger \& Busby 1987, Passos \& Sazima 1995, Castro \& Oliveira 2001).

Manettia é um gênero de ervas, epífitas e lianas, possuindo ca. 220 espécies distribuídas por toda América tropical (Robbrecht 1988, Solomon 2003). É tido como um gênero tipicamente heterostílico possuindo flores geralmente vistosas e polinizadas por beija-flores (Ganders 1979, Passos \& Sazima 1995, Robbrecht 1988). Manettia cordifolia é uma planta trepadeira com os indivíduos dispostos em agrupamentos e florescendo abundantemente (sensu Gentry 1974). É encontrada desde a Argentina (Jujuy), Paraguai, Peru, Bolívia e Brasil (estado do Paraná ao Ceará) (Solomon 2003 e material botânico depositado no herbário da Universidade de Brasília - UB), sendo uma espécie comum nas matas da região de Uberlândia, Minas Gerais. Em estudos prévios na região, foi observado que a espécie não apresentava distilia, apesar de terem sido observadas variações nos tamanho das partes florais.

O presente trabalho tem como objetivo estudar a biologia floral da espécie, avaliando seu sistema de reprodução, morfologia floral e o comportamento de seus polinizadores.

\section{Material e métodos}

Locais de estudo - O trabalho de campo foi realizado de março a agosto de 2000, 2001 e 2003, em duas áreas localizadas no município de Uberlândia, MG: mata de galeria da Estação Ecológica do Panga (EEP) e remanescentes da mata ciliar do Rio Uberabinha [dois locais: Clube de Caça e Pesca Itororó de Uberlândia (CP) e margem do rio localizado no perímetro urbano (MR)]. AEEP está situada a $30 \mathrm{~km}$ do centro da cidade

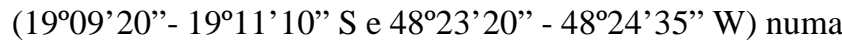
altitude média de $800 \mathrm{~m}$ (Schiavini \& Araújo 1989). O CP situa-se a 8 km oeste do perímetro urbano (18 $8^{\circ} 57^{\prime} \mathrm{S}$ e $48^{\circ} 12^{\prime} \mathrm{W}$ ), enquanto o MR localiza-se ao lado do Clube de recreação "Praia Clube de Uberlândia" (1855'10" S e 48 18'09" W). O clima da região é Aw megatérmico (segundo classificação de Köppen), com duas estações definidas (Rosa et al. 1991). Biologia floral e visitantes - Botões foram marcados antes da abertura e observados para determinar o horário de antese (início da separação das pétalas), longevidade floral (estimada a partir do murchamento da corola) e liberação de pólen. A receptividade estigmática foi determinada a partir da separação dos lobos estigmáticos, uma indicação usualmente utilizada para tal verificação em Rubiaceae (Coelho \& Barbosa 2003), sendo posteriormente confirmada por polinizações manuais e análise do crescimento dos tubos polínicos. Foi utilizada a técnica de coloração com carmim acético para verificar a viabilidade dos grãos de pólen (flores, $\mathrm{n}=4$ ) (Radford et al . 1974). A determinação do número de flores abertas foi realizada no período em que todos os indivíduos das populações estudadas apresentavam-se floridos (máximo de floração).

Em flores ensacadas em pré-antese $(n=20)$ a produção absoluta de néctar foi quantificada usando capilares para medida de volume e refratômetro de mão para concentração de açúcares (sensu Inouye et al. 1980). Por problemas logísticos, as medidas foram realizadas apenas no final do período da manhã (11:00 h). A recompensa calórica por flor foi estimada a partir de métodos descritos em Kearns \& Inouye (1993).

Os visitantes e seu comportamento nas flores foram observados das 6:00 às 17:00 h, totalizando $45 \mathrm{~h}$, no decorrer de seis dias não contínuos. Os polinizadores da espécie foram determinados com base na morfologia dos visitantes e das flores, freqüência de visitas e comportamento dos visitantes. Documentação fotográfica das visitas foi obtida para alguns dos principais polinizadores. Para verificar diferenças na formação de frutos entre flores intactas e flores que sofreram ação de pilhadores foi utilizado o teste de Qui-quadrado 
usando o programa Systat.

Morfometria floral e sistema reprodutivo - Observações sobre a morfologia floral de Manettia cordifolia foram realizadas em flores no campo $(n=35)$ e em material fixado em álcool $70 \%$ (110 flores, sendo cinco flores por indivíduo distribuídas em 22 plantas). Foram tomadas medidas de: comprimento do tubo, abertura, largura mínima e máxima da corola, comprimento da antera e altura do estigma e da antera (da base do estilete ao ápice do estigma e da base da corola ao ápice da antera). Essas medidas foram feitas com auxílio de paquímetro com precisão de $0,5 \mathrm{~mm}$. Dentro de cada população foram feitas, separadamente, comparações entre os indivíduos utilizando ANOVA, sendo o mesmo procedimento utilizado para comparações entre as populações (EEP, 12 indivíduos; MR, cinco; $\mathrm{CP}$, cinco). Todas as análises estatísticas foram conduzidas usando o programa Systat.

Foram analisadas exsicatas da espécie em herbários, com o intuito de verificar a posição das anteras e dos estigmas em flores de outras populações, visando compará-las com as populações estudadas. Todas os exemplares da espécie presentes nos herbários foram analisados, sendo 15 no herbário da Universidade Estadual de Campinas (UEC), 53 no herbário da Universidade de Brasília (UB) e dois no herbário da Universidade Federal de Uberlândia (HUFU).

Testes para definição do sistema de reprodução foram feitos utilizando dez indivíduos, sendo seis na EEP e quatro na MR, tomando cuidado para que cada indivíduo contivesse os diferentes tipos de tratamentos em quantidades semelhantes. Os botões em pré-antese foram ensacados com sacos de organza fina de nylon. No dia da antese foram realizados testes de autopolinização e polinização cruzada manual e preparadas flores para autopolinização espontânea (nos respectivos testes as flores foram posteriormente ensacadas), apomixia (botões emasculados) e polinizações controle (flores expostas). As polinizações cruzadas manuais foram feitas utilizando indivíduos doadores de pólen da mesma área e doadores de pólen de outras áreas.

Algumas flores submetidas aos testes de autopolinização $(n=53)$ e polinização cruzada $(n=48)$ foram coletadas $12,24,48$ e 60 horas após o tratamento, sendo fixadas em FAA 50\% e posteriormente armazenadas em álcool $70 \%$ para preparação de lâminas e observação do crescimento de tubos polínicos em microscopia de fluorescência (Martin 1959).

\section{Resultados}

Biologia floral e visitantes - As flores de Manettia cordifolia são solitárias, pendentes, tetrâmeras, gamossépalas e gamopétalas, com um disco nectarífero na base do tubo da corola. São hermafroditas, com quatro estames epipétalos e as anteras posicionadas na entrada da corola. $\mathrm{O}$ estilete projeta-se acima das anteras nas flores abertas e o conjunto estilete-estigma fica exposto para fora da entrada da corola (figura 1A). Apresenta ovário ínfero, bicarpelar com média de $70 \pm 13,83$ óvulos $(\mathrm{n}=27)$. A antese é diurna, ocorrendo a abertura das flores entre 5:30 e 6:30 h. As flores têm longevidade funcional de até três dias, podendo a corola permanecer murcha (seca) até a formação do fruto maduro. No máximo de floração, os indivíduos apresentam 4,81 $\pm 0,89$ flores abertas $(n=28)$.

$\mathrm{O}$ pólen começa a ser liberado antes da abertura da flor, permanecendo exposto na superfície da antera. A viabilidade polínica estimada foi de $99 \%$ para as flores examinadas (100 grãos de pólen por flor). A receptividade estigmática foi inferida pela morfologia característica do estigma: a partir do botão em préantese, o estigma bífido já se apresentava aberto indicando receptividade.

A recompensa disponível para os visitantes foi o néctar, produzido no disco nectarífero em todos os dias de duração da flor acumulando-se na base do tubo da corola. Flores ensacadas produziram $9,1 \pm 1,3 \mu \mathrm{L}$ de néctar, com concentração de açúcares de 23,2 $\pm 0,97 \%$ e recompensa calórica de ca. 9,2 calorias por flor.

Manettia cordifolia foi regularmente visitada e polinizada pelo beija-flor Phaethornis pretrei Lesson $\&$ DeLattre (1839), que durante visita às flores contactava as partes reprodutivas com a porção frontal da cabeça e com a base da mandíbula (figura 1B). Phaetornis pretrei apresentou comportamento de visita estereotipado, aproximando-se das manchas de recursos por uma direção previsível, emitindo vocalizações e seguindo uma mesma rota dentro da própria mancha. O tempo médio de permanência na mancha foi de $9,96 \pm 10,83 \mathrm{~s}(\mathrm{n}=50)$ variando de três a $60 \mathrm{~s}$, visitando em média 4,6 $\pm 6,52$ flores $(n=50)$ por indivíduo. Em alguns casos isolados, os beija-flores alteraram a rota de visita dentro da mancha visitando muitas flores numa mesma sessão (e.g. 29 flores visitadas), mas voltavam a visitar o mesmo grupo de flores após essas alterações. Phaetornis pretrei visitou uma mesma mancha cerca de quatro vezes por hora, do amanhecer até o final da tarde. Duas outras espécies de beija-flores, Amazilia sp. e Eupetomena macroura J.F. Gmelin (1788), foram registradas visitando flores de $M$. cordifolia, com apenas uma visita para cada espécie.

Três espécies de borboletas, sendo uma Heliconius sp., também visitaram flores de $M$. cordifolia. Elas pousaram defronte à corola e inseriam suas probóscides para alcançar o néctar, ocasionalmente contatando o estigma.

As abelhas Trigona spinipes Fabr. (1875) e Xylocopa sp. foram observadas pilhando néctar por meio 

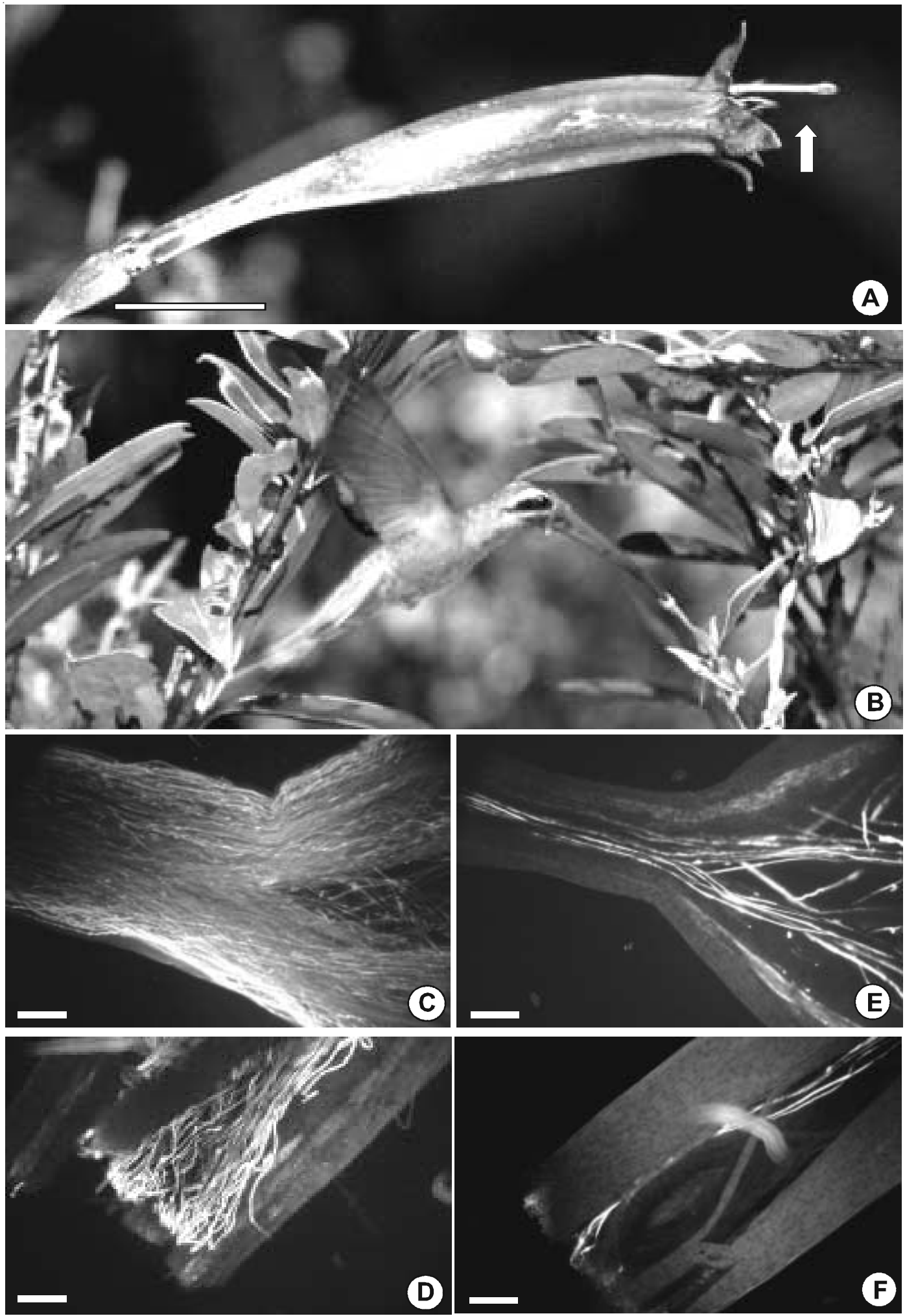

Figura 1. Aspectos morfológicos e polinização de Manettia cordifolia Mart. (Rubiaceae). A. Visão lateral da flor com o complexo estilete/estigma projetando-se além da corola (seta), mostrando a marcante separação estigma-antera ("approach herkogamy"). B. Phaetornis pretrei durante visita à flor de M. cordifolia. C, D. Crescimento de tubos polínicos de polinizações cruzadas após 60 h, início e base do estilete, respectivamente. E, F. Crescimento de tubos polínicos de autopolinizações após $48 \mathrm{~h}$, início e base do estilete, respectivamente. Escala = $1 \mathrm{~cm}(\mathrm{~A}) ; 500 \mu \mathrm{m}(\mathrm{C}, \mathrm{E}) ; 250 \mu \mathrm{m}(\mathrm{D}, \mathrm{F})$. 
de perfurações na base da corola. Além do néctar, T. spinipes foi registrada pilhando pólen, sem contactar o estigma.

Outra espécie pilhadora de néctar foi a ave Coereba flaveola Linnaeus (1758). Ela apoiava-se nas flores e perfurava a corola ou utilizava as perfurações feitas por T. spinipes para se alimentar. A pilhagem não impediu, posteriormente, visitas legítimas de $P$. pretrei, pois mesmo após pilhagem de néctar, houve formação de frutos após polinização natural, não havendo diferença significativa na formação de frutos entre flores intactas e flores que sofreram ação de pilhadores $\left(\chi^{2}=0,64 ; \mathrm{gl}=1 ; \mathrm{p}=0,05\right)$.

Monomorfismo e sistema reprodutivo - Manettia cordifolia apresentou flores com estigma acima do nível das anteras, havendo separação espacial entre os órgãos (separação estigma-antera; figura 1A). A espécie apresentou ainda separação estigma-antera com distribuição unimodal (figura 2) e altura do estigma e das anteras com distribuição contínua entre as plantas. Entretanto, a separação estigma-antera variou tanto entre flores do mesmo indivíduo quanto entre indivíduos. Excepcionalmente, em duas flores de indivíduos diferentes, o estigma estava localizado abaixo das anteras $(0,3$ e $0,5 \mathrm{~mm})$.

Em todas as exsicatas verificadas nos herbários, foi observado que o estigma estava localizado sempre acima do nível das anteras. Esses exemplares eram provenientes de populações dos estados do Ceará e Mato Grosso do Sul com uma exsicata cada, Mato Grosso duas, Paraná quatro, Goiás 11, Bahia 13, São Paulo 18 e Minas Gerais 20. Além das exsicatas, uma população estudada por A.V. Lopes (dados não publicados) no estado de Pernambuco também apresentou tal característica.

Tanto entre os indivíduos de cada área, como entre as populações estudadas (EEP, CP e MR), as alturas do estigma e das anteras apresentaram diferenças significativas. Os dados morfométricos variaram entre as populações, sendo que não houve diferença significativa apenas para a largura mínima da corola. $\mathrm{Na}$ análise dos indivíduos separadamente para cada população, diferenças significativas ocorreram entre as plantas da mesma localidade, entretanto as diferenças

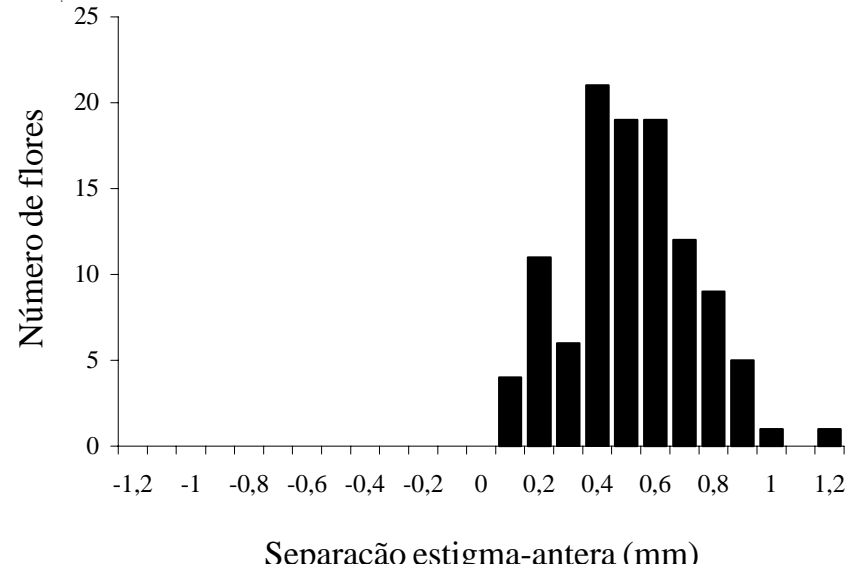

Figura 2. Distribuição unimodal da separação estigma-antera em flores de Manettia cordifolia Mart. (Rubiaceae) indicando monomorfismo longistílico na espécie.

Figure 2. Unimodal distribution of stigma-anther separation in flowers of Manettia cordifolia Mart. (Rubiaceae) showing pin-monomorphism in the species.

não foram as mesmas para cada população. Na EEP, não ocorreu diferença significativa apenas para a abertura da corola, enquanto no $\mathrm{CP}$ não houve diferença somente para a largura máxima e para a abertura da corola, e, por fim, na MR as diferenças não aconteceram na abertura e no comprimento do tubo da corola (tabela 1).

Os testes de polinização controlada indicam que a espécie é auto-incompatível e não agamospérmica (tabela 2). Nas polinizações cruzadas, os grãos de pólen foram observados germinando no estigma após $12 \mathrm{~h}$, com um grande número de tubos polínicos alcançando o primeiro quarto do estilete (sete pistilos em 10 observados, tendo os outros três pistilos um pequeno número de tubos polínicos alcançando o primeiro quarto). Após $24 \mathrm{~h}$, foram constatados feixes de tubos polínicos crescendo em grande quantidade até aproximadamente a metade do estilete (12 pistilos em 14 , tendo os outros dois pistilos pequena quantidade de tubos polínicos na porção mediana). Em 48 h, os tubos polínicos foram observados chegando no final do estilete e entrando no ovário (10 em 12 pistilos, enquanto nos outros dois pistilos não foram observados tubos polínicos alcançando a parte

Figure 1. Manettia cordifolia Mart. (Rubiaceae), at the "Estação Ecológica do Panga", Uberlândia, MG, Brazil. A. Lateral view of the flower with the complex style/stigma projected beyond the corolla (arrow), showing notable stigma-anther separation (approach herkogamy). B. Phaetornis pretrei during a visit to M. cordifolia flower. C., D. Cross pollen tube growth $60 \mathrm{~h}$ after pollination, respectively tip and style base. E., F. Self- pollen tube growth $48 \mathrm{~h}$ after pollination, respectively tip and style base. Scale bar $=1 \mathrm{~cm}(\mathrm{~A}) ; 500 \mu \mathrm{m}(\mathrm{C}, \mathrm{E}) ; 250 \mu \mathrm{m}(\mathrm{D}, \mathrm{F})$. 
Tabela 1. Medidas das características florais de Manettia cordifolia Mart. (Rubiaceae) (cm), com suas respectivas médias e desvios padrão (SD) para todas as flores (110) e separadamente para cada população (EEP = Estação Ecológica do Panga, Uberlândia, MG, Brasil; CP = Reserva Legal do Clube de Caça e Pesca Itororó, Uberlândia, MG, Brasil; MR = Margem do Rio Uberabinha, Uberlândia, MG, Brasil). Resultados das análises estatísticas (ANOVA) feitas para comparação entre populações e entre indivíduos de cada população. * = Significativamente diferente $(\mathrm{p}<0,05)$.

Table 1. Measurements of floral characteristics of Manettia cordifolia Mart. (Rubiaceae) (cm), with mean value and standard deviation (SD) calculated for all flowers (110) and for each population (EEP = "Estação Ecológica do Panga", Uberlândia, Minas Gerais, Brazil; CP = "Reserva Legal do Clube de Caça e Pesca Itororó", Uberlândia, Minas Gerais, Brazil; MR = "Margem do Rio Uberabinha", Uberlândia, Minas Gerais, Brazil). Results of the statistical analysis (ANOVA) for comparison between populations and between individuals of each population. * = Significantly different $(\mathrm{p}<0,05)$.

\begin{tabular}{|c|c|c|c|c|c|c|c|c|}
\hline \multirow{3}{*}{ Medidas florais } & \multicolumn{4}{|c|}{ Medidas florais } & \multicolumn{4}{|c|}{ Análise estatística } \\
\hline & \multirow{2}{*}{$\frac{\text { Flores }}{\text { Média total } \pm \text { SD }}$} & \multicolumn{3}{|c|}{$\begin{array}{l}\text { População (n. de flores) } \\
\text { Média } \pm \text { SD/população }\end{array}$} & \multirow{2}{*}{$\begin{array}{l}\text { Populações } \\
\frac{F}{\mathrm{EEP} / \mathrm{CP} / \mathrm{MR}}\end{array}$} & \multicolumn{3}{|c|}{$\begin{array}{l}\text { Indivíduos } \\
\mathrm{F}\end{array}$} \\
\hline & & $\operatorname{EEP}(55)$ & $\mathrm{CP}(30)$ & MR (25) & & EEP & $\mathrm{CP}$ & MR \\
\hline Altura do estigma & $5,24 \pm 0,52$ & $5,42 \pm 0,6$ & $5,14 \pm 0,41$ & $4,97 \pm 0,23$ & $8,02 *$ & $8,19 *$ & $7,91 *$ & $4,81^{*}$ \\
\hline Altura da antera & $4,79 \pm 0,5$ & $4,99 \pm 0,55$ & $4,67 \pm 0,43$ & $4,49 \pm 0,22$ & $11,17 *$ & $7,93 *$ & $12,06 *$ & $5,47^{*}$ \\
\hline Comprimento da corola & $4,31 \pm 0,45$ & $4,46 \pm 0,48$ & $4,27 \pm 0,43$ & $4,04 \pm 0,24$ & $8,4 *$ & $10,51 *$ & $15,73 *$ & 2,22 \\
\hline Abertura da corola & $0,58 \pm 0,08$ & $0,6 \pm 0,08$ & $0,5 \pm 0,09$ & $0,59 \pm 0,06$ & $5,26 *$ & 1,65 & 2,47 & 1,64 \\
\hline Largura mínima da corola & $0,2 \pm 0,02$ & $0,21 \pm 0,02$ & $0,2 \pm 0,02$ & $0,2 \pm 0,02$ & 1,94 & $3,05^{*}$ & $5,7^{*}$ & $3,11 *$ \\
\hline Largura máxima da corola & $0,63 \pm 0,08$ & $0,65 \pm 0,09$ & $0,6 \pm 0,08$ & $0,59 \pm 0,06$ & $4,5^{*}$ & $5,42 *$ & 2,36 & $3,69 *$ \\
\hline Comprimento da antera & $0,5 \pm 0,05$ & $0,53 \pm 0,04$ & $0,49 \pm 0,05$ & $0,45 \pm 0,04$ & $20,5^{*}$ & $10,07 *$ & $5,88^{*}$ & $5,84 *$ \\
\hline
\end{tabular}

final do estilete). Após $60 \mathrm{~h}$, todos os pistilos observados tiveram grande quantidade de tubos polínicos chegando no final do estilete (figura 1C, D), entrando no ovário e penetrando nos óvulos $(\mathrm{n}=12)$.

Para autopolinização, após 12 h, também foram observados grãos de pólen germinando no estigma, todavia poucos alcançando o primeiro quarto do estilete (dois pistilos em 10, tendo os outros oito nenhum registro de alcance). Em $24 \mathrm{~h}$, poucos tubos polínicos foram observados crescendo até aproximadamente metade do estilete, tendo, os que alcançaram, a aparência frágil (menor diâmetro e menos fluorescência que os tubos polínicos de polinização cruzada) (12 em 17 , tendo os outros cinco também pequena quantidade, mas com aparência semelhante aos de polinização cruzada). Após $48 \mathrm{~h}$, poucos tubos polínicos foram observados no início e na base do estilete (figura $1 \mathrm{E}, \mathrm{F}$; três em 15 pistilos, com os outros 12 pistilos não havendo registro de penetração no ovário). Por fim, após 60 h, a ausência de tubos polínicos penetrando nos óvulos foi notável (10 em 11 pistilos), havendo apenas um pistilo com registro duvidoso de penetração. Ainda em autopolinização, observando-se de um modo geral todos os pistilos tratados, notou-se uma diminuição substancial no número

Tabela 2. Resultados dos tratamentos de polinizações controladas em Manettia cordifolia Mart. (Rubiaceae).

Table 2. Results of hand pollination treatments in Manettia cordifolia Mart. (Rubiaceae).

\begin{tabular}{lccc}
\hline Tratamento & $\begin{array}{c}\text { Número de flores } \\
\text { polinizadas }\end{array}$ & $\begin{array}{c}\text { Número de frutos } \\
\text { formados }\end{array}$ & $\begin{array}{c}\text { Sucesso } \\
\text { reprodutivo (\%) }\end{array}$ \\
\hline Autopolinização manual & 102 & 4 & 4 \\
Autopolinização espontânea & 50 & 2 & 4 \\
Agamospermia & 50 & 0 & 0 \\
Polinização cruzada & 108 & 25 & 23 \\
Polinização natural (controle) & 50 & 9 & 18 \\
Polinização natural ${ }^{(1)}$ & 50 & 7 & 14 \\
\hline
\end{tabular}

${ }_{1}^{1}$ perfuração da corola e pilhagem de néctar causadas por Trigona spinipes (Apidae) e Coereba flaveola (Fringillidae). 
de tubos polínicos que crescem no início do estilete, chegando em sua porção final.

\section{Discussão}

Biologia floral e visitantes - A polinização por beijaflores pode ser considerada freqüente em Rubiaceae, já que várias de suas espécies dependem dessas aves para a polinização (Bawa \& Beach 1983, Passos \& Sazima 1995, Castro \& Oliveira 2001). Manettia cordifolia apresenta características típicas da síndrome ornitofilia, tais como antese diurna, corola tubular relativamente rígida, coloração vermelha, néctar abundante localizando-se distante dos órgãos sexuais e ausência de odor (Faegri \& Van der Pijl 1979). Nesse caso em particular, ao contrário do que tem sido evidenciado para outras plantas (Waser et al. 1996), a síndrome de polinização da espécie funciona como um bom indicador do seu principal tipo de polinizador.

A longevidade das flores de $M$. cordifolia (três dias) é maior que a longevidade média (1,37 dias) registrada para espécies de Rubiaceae (Stratton 1989), todavia assemelha-se em partes à de M. luteo-rubra Benth. (quatro dias; Passos \& Sazima 1995). Essa grande longevidade pode aumentar as chances de visitação e polinização cruzada (Primack 1985, Feinsinger \& Busby 1987, Stratton 1989, Passos \& Sazima 1995). Tal característica pode estar associada a espécies ornitófilas (Passos \& Sazima 1995, Castro \& Oliveira 2001), pois sua menor freqüência de visitas em relação às espécies entomofílicas faz com que uma maior longevidade aumente as chances de contato com os órgãos reprodutivos (Stone 1996).

Os valores de volume de néctar, concentração de açúcares e recompensa energética são, até certo ponto, semelhantes aos de outras espécies polinizadas por beijaflores (e.g. Baker 1975, Passos \& Sazima 1995). Os dados obtidos ao final da manhã para $M$. cordifolia são próximos àqueles de $M$. luteo-rubra, que produz $8,46 \mu \mathrm{L} \pm 3,68$ de néctar, com concentração de $24 \% \pm 2,11$ (Passos \& Sazima 1995), e ca. de 8,9 calorias por flor. Por essas espécies possuírem características da síndrome ornitofilia, é possível enquadrá-las na categorização proposta por Feinsinger \& Colwell (1978) para flores visitadas por beija-flores, baseada nas características dos recursos oferecidos (valores de néctar e distribuição das flores). Segundo essa categorização, M. cordifolia e M. luteo-rubra poderiam ser definidas como flores agrupadas com moderada recompensa energética ("clumped moderate flowers"). Entretanto, Passos \& Sazima (1995) classificaram $M$. luteo-rubra como flores agrupadas com rica recompensa energética ("clumped rich flowers"). Tal categorização parece equivocada, pois a recompensa energética oferecida por flores desta categoria, no trabalho de Feinsinger \& Colwell (1978), é algo da ordem de dez vezes maior que a oferecida pelas flores de ambas as espécies de Manettia. É possível que o número de flores oferecido por planta tenha sido muito maior em $M$. luteo-rubra, criando agrupamentos particularmente ricos e atrativos, mas a recompensa energética por flor oferecida por ambas espécies parece ser apenas moderada ("clumped moderate flowers").

Manettia cordifolia teve como principal polinizador Phaetornis pretrei. Esse beija-flor possui o bico longo e levemente curvado (ca. 33 mm; Piratelli 1997, Castro \& Oliveira 2001), encaixando-se perfeitamente na flor de $M$. cordifolia (comprimento do tubo da corola com ca. $43 \pm 0,456 \mathrm{~mm}$ ), aumentando, possivelmente, a eficiência na transferência dos grãos de pólen. Espécies de Phaetornis parecem forragear em linhas de captura com alta recompensa energética ("high-reward trapliners") visitando flores espalhadas com grande quantidade de néctar, de 69 a $120 \mu \mathrm{L}$ com 98-169 cal (Feinsinger \& Colwell 1978). Entretanto, M. cordifolia oferece flores agrupadas com moderada quantidade de néctar e energia, fazendo com que $P$. pretrei forrageie em linhas de captura de baixa recompensa energética, "low-reward trapliners". Dadas estas limitações energéticas, é provável que o beija-flor seja forçado a visitar um número bem maior de flores para suprir suas necessidades, aumentando, conseqüentemente seu deslocamento entre as plantas e proporcionando maiores taxas de polinização cruzada. Em M. luteo-rubra, talvez pela concentração de recursos em manchas mais ricas, Phaetornis eurynome e P. squalidus comportaram-se como territorialistas (Passos \& Sazima 1995). Nessa planta, o comprimento menor do tubo da corola $(16,19 \pm 1,68 \mathrm{~mm}$ para flores brevistilas e $14,75 \pm 1,73 \mathrm{~mm}$ para longistilas) possibilitou a exploração de néctar por beija-flores com diversos tamanhos de bicos, inclusive Thalurania glaucopis, um Trochilinae de bico curto. A corola mais longa em M. cordifolia restringe as visitas principalmente de beija-flores com bico mais curto.

Os outros visitantes florais foram menos importantes. Os lepidópteros que visitaram $M$. cordifolia podem funcionar como polinizadores secundários, já que espécies de Heliconius podem utilizar, inclusive, estratégia de forrageamento "trapline" (Ehrlich \& Gilbert 1973). 
Os pilhadores T. spinipes, Xylocopa sp. e Coereba flaveola aparentemente tiveram efeito neutro no sucesso reprodutivo da espécie, já que não influenciaram o comportamento do polinizador principal. Phaetornis pretrei continuou visitando flores pilhadas nas quais formaram-se frutos em taxas similares àquelas não pilhadas após polinização natural.

Monomorfismo e sistema reprodutivo - $\mathrm{O}$ gênero Manettia é tido como tipicamente heterostílico (Ganders 1979), sendo tal situação encontrada em M. luteo-rubra (Passos \& Sazima 1995). Entretanto, existem espécies dentro de gêneros majoritariamente distílicos que apresentam variações nas características florais, como é o caso de $M$. cordifolia. Isso pode ocorrer em conseqüência de alterações no sistema de reprodução, podendo ser variável dependendo das características florais da população, demografia local, situações ecológicas, condições ambientais e taxas de recombinação gênica (Sobrevila et al. 1983, Barrett \& Shore 1987, Thompson 2001, Endels et al. 2002).

Em $M$. cordifolia, a distribuição unimodal na separação estigma-antera e a distribuição contínua da altura do estigma e da antera entre os indivíduos amostrados, indicam que a espécie não é distílica. As espécies são consideradas heterostílicas quando os indivíduos podem ser divididos em duas classes morfológicas distintas (longistilas e brevistilas) com uma severa hercogamia recíproca entre os morfos (Faivre $\&$ McDade 2001). Com base nessas condições estruturais, a espécie apresentou características morfológicas de monomorfismo longistílico (Barrett 1992, Hamilton 1990) e "approach herkogamy" (disposição morfológica do complexo estigma-antera, com o estigma localizando-se sempre acima das anteras). O monomorfismo é caracterizado quando apenas um dos morfos é encontrado, podendo esse ser longistilo ou brevistilo (Ganders 1979), enquanto flores que apresentam anteras e estigma no mesmo nível são denominadas de homostílicas (Hamilton 1990). Esses polimorfismos possuem, de certa forma, uma ligação, pois ambos, provavelmente evoluíram a partir da distilia (Hamilton 1990, Pailler \& Thompson 1997). Barrett \& Shore (1987) argumentam que o monomorfismo é originado da distilia, todavia passando primeiramente pela condição homostílica autocompatível. Porém, outros autores defendem que o monomorfismo pode ser derivado diretamente da condição distílica, sem a passagem pela condição homostílica (Baker 1966, Nicholls 1985, Hamilton 1990).

A condição de $M$. cordifolia, como monomórfica auto-incompatível, pode ter evoluído de diversas formas.
Devido às características relativamente especializadas da flor, a hipótese da espécie representar uma condição original de "approach hercogamy" dentro do gênero pode ser tratada com reservas, mas estudos filogenéticos mais específicos seriam necessários antes de se afastar em definitivo tal alternativa. Considerando que esta é uma condição de monomorfismo longistílico derivada da distilia, é possível que, com o desaparecimento do morfo oposto e a permanência da auto-incompatibilidade, o surgimento da compatibilidade intramorfo atual tenha sido necessária para manter o sucesso reprodutivo da espécie (Hamilton 1990). Essa característica pode ter surgido pela perda ou relaxamento da capacidade de inibição do crescimento do tubo polínico devido a um "cross-over" ou uma quebra de ligação no supergene, em que as características morfológicas longistílicas foram mantidas, mas a regulação do sistema de incompatibilidade tornouse independente (Baker 1966, Nicholls 1985). Espécies como Palicourea petiolaris Aubl. (Sobrevila et al. 1983), Hamelia patens Jacq., H. xerocarpa Kuntze, Posoqueria grandiflora $\mathrm{H}$. Karst. e Warszewiczia coccinea (Vahl) Klotzsch (Bawa \& Beach 1983) possuem as mesmas características morfológicas e as mesmas condições de incompatibilidade de $M$. cordifolia, sendo a origem destas características explicadas de maneira similar. Outra possibilidade seria a de que, em decorrência de falhas naturais no sistema de incompatibilidade comuns no morfo longistilo (Hamilton 1990), frutos e sementes formados por polinizações intramorfo fossem suficientes para manter o sucesso reprodutivo e a manutenção da espécie.

A prevalência morfológica do monomorfismo longistílico em espécies derivadas de ancestrais distílicos pode ocorrer pelo fato deste morfo ter vantagens seletivas sobre o brevistilo (Philipp \& Schou 1981). Um suporte a esta idéia seria a prevalência, nas Angiospermas, de "approach herkogamy" em relação a hercogamia reversa (Barrett 1992). O posicionamento exserto do estigma facilitaria o contato com o grão de pólen presente no polinizador (Nicholls 1985, Dulberger 1992). Em Hedyotis caerulea (L.) Hook., houve grande fluxo de pólen intramorfo, principalmente entre flores longistilas (Ornduff 1980). Flores longistilas de Psychotria birotula Sm. \& Downs e P. pubigera Schlecht. foram mais freqüentes do que flores brevistilas, indicando uma assimetria no fluxo do pólen em direção ao morfo longistílico (Castro \& Oliveira 2002). Alterações na proporção de transferência de grãos de pólen e prevalência no estabelecimento de um morfo podem estar associados também a distúrbios ecológicos na área em que se encontra a espécie, tais como 
perturbação ambiental, isolamento geográfico, interação com polinizadores e variações nas características florais (Sobrevila et al. 1983, Endels et al. 2002). Todas essas condições podem favorecer o estabelecimento e a permanência de apenas um morfo na espécie havendo a perda do morfo oposto sem prejuízo para o sucesso reprodutivo da espécie (Martin 1967, Nicholls 1985).

O monomorfismo floral em Manettia cordifolia está associado com a auto-incompatibilidade, como registrado também em Hamelia patens Jacq., H. xerocarpa Kuntze Posoqueria grandiflora Standl. e Warszewiczia coccinea Klotzsch (Bawa \& Beach 1983), todavia não foram evidenciadas barreiras de incompatibilidade bem definidas. A inibição total dos tubos polínicos de autopolinização não aconteceu em uma região exata, e os que passaram do estigma foram interrompidos ao longo do estilete; poucos alcançaram o ovário e, em apenas um caso, ocorreu penetração nos óvulos. Situação similar foi encontrada em polinizações entre os morfos longistilos de M. luteo-rubra (Passos \& Sazima 1995) e Psychotria chiapensis Standl. (Bawa \& Beach 1983). Essas características de crescimento de tubos polínicos são similares às observadas em plantas com sistema de incompatibilidade gametofítico, entretanto nas Rubiaceae e nas plantas distílicas de uma maneira geral, parecem ser comuns os sistemas esporofíticos (Bawa \& Beach 1983). Sistemas de reprodução heteromórficos incluem uma diversidade de mecanismos de reconhecimento e rejeição de tubos polínicos, sendo ainda pouco conhecidos nas Rubiaceae, onde, possivelmente, existem dois ou mais mecanismos de incompatibilidade (Bawa \& Beach 1983).

Apesar de incluída num gênero tipicamente distílico, Manettia cordifolia pode ser considerada uma espécie monostílica, já que os dados obtidos das populações estudadas e das várias exsicatas avaliadas, provenientes de outras áreas, mostraram tal situação. Entretanto, dizer precisamente qual(is) força(s) seletiva(s) direcionaram tal condição ainda não é possível, pois alterações nas características heterostílicas podem ter diversas explicações. Informações sobre a distribuição geográfica e a filogenia do grupo poderão ajudar a entender melhor a situação da espécie e o surgimento dessas características monostílicas.

Agradecimentos - Ao Dr. Peter Gibbs e às Dras. Isabel Cristina Machado, Solange Cristina Augusto e Celine de Melo pelas sugestões na elaboração do manuscrito. Aos herbários UEC, HUFU e UnB pela permissão para a análise do material botânico. À Capes pela bolsa de mestrado para o primeiro autor e ao CNPq pelo apoio financeiro no âmbito do projeto integrado 520872/96-7.

\section{Referências bibliográficas}

BAKER, H.G. 1966. The evolution, functioning and breakdown of heteromorphic incompatibility systems I. The Plumbaginaceae. Evolution 18:507-512.

BAKER, H.G. 1975. Sugar concentrations in nectars from hummingbird flowers. Biotropica 7:37-41.

BARRETT, S.C.H. 1992. Heterostylous genetic polymorphisms: model systems for evolutionary analysis. In Evolution and function of heterostyly (S.C.H Barrett, ed.). Monographs on theorical and applied genetics. Springer-Verlag, Berlin, p.1-24.

BARRETT, S.C.H. \& SHORE, J.S. 1987. Variation and evolution of breeding systems in Turnera ulmifolia L. complex (Turneraceae). Evolution 41:340-354.

BARRETT, S.C.H. \& RICHARDS, J.H. 1990. Heterostyly in tropical plants. Memoirs of the New York Botanical Garden 55:35-61.

BARRETT, S.C.H., WILKEN, D.H. \& COLE, W.W. 2000. Heterostyly in the Lamiaceae: the case of Salvia brandegeei. Plant Systematics and Evolution 223:211-219.

BAWA, K.S. \& BEACH, J.H. 1983. Self-incompatibility systems in the Rubiaceae of a tropical lowland wet forest. American Journal of Botany 70:1281-1288.

CASTRO, C.C. \& OLIVEIRA, P.E. 2001. Reproductive biology of the protandrous Ferdinandusa speciosa Pohl. (Rubiaceae) in southeastern SE Brazil. Revista Brasileira de Botânica 24:167-172.

CASTRO, C.C. \& OLIVEIRA, P.E. 2002. Pollination biology of distylous Rubiaceae in the Atlantic Rain Forest, SE Brazil. Plant Biology 4:640-646.

COELHO, C.P. \& BARBOSA, A.A. 2003. Biologia reprodutiva de Palicourea macrobotrys Ruiz \& Pavon (Rubiaceae): um possível caso de homostilia no gênero Palicourea Aubl. Revista Brasileira de Botânica 26:403-413.

DULBERGER, R. 1992. Floral polymorphisms and their functional significance in the heterostylous syndrome. In Evolution and function of heterostyly (S.C.H Barrett, ed.). Monographs on theorical and applied genetics. Springer-Verlag, Berlin, p.41-77.

EHRLICH, P.R. \& GILBERT, L.E. 1973. Population structure and dynamics of the tropical butterfly Heliconius ethilla. Biotropica 5:69-82.

ENDELS, P., JACQUEMYN, H., BRYS, R. \& HERMY, M. 2002. Changes in pin-thrum ratios in populations of the heterostyle Primula vulgaris Huds: Does imbalance affect populations persistence? Flora 197:326-331.

FAEGRI, K. \& VAN DER PIJL, L. 1979. The principles of pollination ecology. Pergamon Press, Oxford.

FAIVRE, A.M. \& MCDADE, L.A. 2001. Population-level variation in the expression of heterostyly in three species of Rubiaceae: does reciprocal placement of anthers and stigmas characterize heterostyly? American Journal of Botany 88:841-853. 
FEINSINGER, P. \& COLWELL, R.K. 1978. Community organization among Neotropical nectar-feeding birds. American Zoology 18:779-795.

FEINSINGER, P. \& BUBSY, W.H. 1987. Pollen carryover: experimental comparisons between morphs of Palicourea lasiorrachis (Rubiaceae) a distylous, birdpollinated, tropical treelet. Oecologia 73:231-235.

GANDERS, F.R. 1979. The biology of heterostyly. New Zealand Journal of Botany 17:607-635.

GENTRY, A.H. 1974. Flowering phenology and diversity in tropical Bignoniaceae. Biotropica 6:64-69.

HAMILTON, C.W. 1990. Variations on a distylous theme in Mesoamerican Psychotria subgenus Psychotria (Rubiaceae). Memoirs of the New York Botanical Garden 55:65-75.

IMBERT, F.M. \& RICHARDS, J.H. 1993. Protandry, incompatibility, and secondary pollen presentation in Cephalanthus occidentalis (Rubiaceae). American Journal of Botany 80:395-404.

INOUYE, D.W., FAVRE, N.D., LANUN, J.A., LEVINE, D.M., MEYERS, J.B., ROBERTS, M.S., TSAO, F.C. \& WANG, Y.Y. 1980. The effect of non-sugar nectar constituents on estimates of nectar energy content. Ecology 61:992-995.

KEARNS, C.A. \& INOUYE, D.W. 1993. Techniques for pollination biologists. University Press of Colorado, Niwot.

KOHN, J.R. \& BARRET, S.C.H. 1992. Experimental studies on the functional significance of heterostyly. Evolution 46:43-55.

LI, P. \& JOHNSTON, M.O. 2001. Comparative floral morphometrics of distyly and homostyly in three evolutionary lineages of Amsinckia (Boraginaceae). Canadian Journal of Botany 79:1332-1348.

MARTIN, F.N. 1959. Staining and observing pollen tubes in the style by means of fluorescence. Stain Technology 34:125-128.

MARTIN, F.W. 1967. Distyly, self-incompatibility and evolution in Melochia. Evolution 21:493-499.

NICHOLLS, M.S. 1985. The evolutionary breakdown of distyly in Linum tenuifolium (Linaceae). Plant Systematics and Evolution 150:291-301.

ORNDUFF, R. 1980. Heterostyly, population composition and pollen flow in Hedyotis caerulea. American Journal of Botany 67:95-103.

ORTIZ, P.L., ARISTA, M. \& TAVALERA, S. 2000. Pollination and breeding systems of Putoria calabrica (Rubiaceae), a Mediterranean dwarf shrub. Plant Biology 2:325-330.

PASSOS, L. \& SAZIMA, M. 1995. Reproductive biology of the distylous Manettia luteo-rubra (Rubiaceae). Botanica Acta 108:309-313.

PAILLER, T. \& THOMPSON, J. D. 1997. Distyly and variation in heteromorphic incompatibility in Gaertnera vaginata (Rubiaceae) endemic to La Reunion Island. American Journal of Botany 84: 315-317.

PHILIPP, M. \& SCHOU, O. 1981. An unusual heteromorphic incompatibility system - distyly, self-incompatibility, pollen load and fecundity in Anchusa officinalis (Boraginaceae). New Phytologist 89:693-703.
PIRATELLI, A.J. 1997. Comportamento alimentar de beijaflores em duas espécies de Hippeastrum Herb. (Amaryllidaceae). Revista Brasileira de Biologia 57:261-273.

PRIMACK, R.B. 1985. Longevity of individual flowers. Annual Review of Ecology and Systematics 16:15-37.

RADFORD, A.E., DICIKSON, W.C., MASSEY, J.R. \& BELL, C.R. 1974. Vascular plant systematics. Harper \& Row, New York.

RICHARDS, J.H. \& KOPTUR, S. 1993. Floral variation and distyly in Guettarda scabra (Rubiaceae). American Journal of Botany 80:31-40.

RIVEROS, G.M., BARRIA, O.R. \& HUMANÃ, A.M. 1995. Selfcompatibility in distylous Hedyotis salzmannii (Rubiaceae). Plant Systematics and Evolution 194:1-8.

ROBBRECHT, E. 1988. Tropical woody Rubiaceae. Opera Botanica Belgica 1:1-271.

ROSA, R., LIMA, S.C. \& ASSUNÇÃO, W.L. 1991. Abordagem preliminar das condições climáticas de Uberlândia (MG). Sociedade e Natureza 3:91-108.

SCHIAVINI, I. \& ARAÚJO, G.M. 1989. Considerações sobre a vegetação da Reserva Ecológica do Panga, Uberlândia, MG. Sociedade e Natureza 1:61-66.

SNOW, D.W. \& SNOW, B.K. 1986. Feeding ecology of hummingbirds in the Serra do Mar, Southeastern Brazil. El Hornero 12:286-296.

SOBREVILA, C., RAMIREZ, N. \& ENRECH, N.X. 1983. Reproductive biology of Palicourea fendleri and $P$. petiolares (Rubiaceae), heterostylous shrubs of a tropical cloud forest in Venezuela. Biotropica 15:161-169.

SOLOMON, J. 2003. Missouri Botanical Garden. http:// www.mobot.org (acesso em 10/11/2003).

STONE, J.L. 1996. Components of pollination effectiveness in Psychotria suerrensis, a tropical distylous shrub. Oecologia 107:504-512.

STRATTON, D.A. 1989. Longevity of individual flowers in a Costa Rican cloud forest: ecological correlates and phylogenetic constraints. Biotropica 21:308-318.

TAYLOR, C.M. 1996. Overview of the Psychotrieae (Rubiaceae) in the Neotropics. Opera Botanica Belgica 7:261-270.

THOMPSON, J.D. 2001. How do visitation patterns vary among pollinators in relation to floral display and floral design in generalist pollination system? Oecologia 126: 386-394.

THOMPSON, J.D. \& DOMMÉE, B. 2000. Morph-specific patterns of variation in stigma height in natural populations of distylous Fasminum fruticans. New Phytologist 148:303-314.

WASER, N.M., CHITTKA, L., PRICE, M.V., WILLIAMS, N.M. \& OLLERTON, J. 1996. Generalization in pollination systems, and why it matters. Ecology 77:1043-1060.

WOLFF, D., BRAUN, M. \& LIEDE, S. 2003. Nocturnal versus diurnal pollination success in Isertia laevis (Rubiaceae): A sphingophilous plant visited by hummingbirds. Plant Biology 5:71-78. 